\title{
TRUCOS EN TÉCNICAS LAPAROSCÓPICAS
}

\author{
Antonio Rosales Bordes
}

Servicio de Urología. Fundación Puigvert Barcelona. España.

ÍN DICE/ RESUMEN

Trucos en pacientes con antecedentes de cirugía abdominal previa, en obesos y en caso de lesión de los vasos epigástricos.

A. Rosales Bordes.

Palabras clave: Tecnica laparoscopica. Cirugía abdominal. Pacientes obesos. Lesion vasos epigástricos.
Antonio Rosales Bordes

Servicio de Urología

Fundación Puigvert

Cartagena, 340-350

08025 -Barcelona. (España)
TRUCOS EN EL ABORDAJE EN PACIENTES CON ANTECEDENTES DE CIRUGÍA ABDOMINAL PREVIA, EN OBESOS Y EN CASO DE LESIÓN DE LOS VASOS EPIGÁSTRICOS.

A. Rosales Bordes

Los antecedentes de abordajes quirúrgicos previos no constituyen una contraindicación para la practica de cirugía laparoscópica transperitoneal, aunque si puede dificultar el procedimiento. Uno de los detalles principales a la hora de colocar los trocares es que el inicial debe posicionarse mediante visión directa ya sea a través de minilaparotomía o mediante distintos dispositivos que se fundamentan en trocares trasparentes de $5012 \mathrm{~mm}$ que permiten la entrada de una óptica laparoscópica de 0 grados que permite el control de los distintos planos de la pared abdominal hasta llegar a la cavidad peritoneal. Tanto Ethicon ${ }^{\circledR}$ como Tyco ${ }^{\circledR}$ presentan distintos dispositivos en su haber ( 0 ptiview y Visiport), que difieren en que uno presenta una punta con dos extremos a filados que progresa por la pared abdominal mediante la presión de la mano del cirujano y otro posee una pequeña cuchilla que se libera mediante un dispositivo de gatillo que secciona de forma "controlada" los distintos planos de la pared abdominal. 0 tras casas comerciales ofrecen trócares metálicos en forma de "sacacorchos" que permiten la introducción de una óptica de 0 은 a travesando las distintas capas de la pared abdominal bajo visión endoscópica.

De todas maneras nosotros preferimos la práctica de una incisión de 2 cms para la colocación del primer trocar en una región alejada de la o las 
cicatrices abdominales previas. La a pertura por planos nos facilitará la llegada al peritoneo y nos permitirá liberar las adherencias del epiplón o del intestino que se encuentren subyacentes. Una disección digital roma nos permitirá la colocación del trocar e iniciar la insuflación. Tras esta maniobra se puede colocar un trocar de Hasson o como en nuestro medio un trocar transparente para dejarlo bajo visión en la posición más correcta colocando su extremo a 2-3 cms del peritoneo parietal. Es conveniente para evitar la movilización del trocar de la óptica durante los distintos pasos de la intervención, la colocación de un punto de cierre en la incisión cutánea mediante un punto de seda o la colocación de un drenaje de Penrose enrollado en la base del trocar y fijado mediante un mosquito.

La insuflación ha de iniciarse lentamente a un ritmo de 5 litros/ minuto. Si el marcador del insuflador va aumentando lentamente hasta la cifra de presión intrabdominal prefijado (13-15 mm de $\mathrm{Hg}$.) es que la distribución del gas se realiza uniformemente. La elevación brusca y mantenida de la presión refleja una cavidad abdominal tabicada o la situación del trocar en el seno de una adherencia epiploica o en íntimo contacto con asas intestinales. La introducción de la óptica nos orientara sobre como se encuentra el "terreno quirúrgico" y si contamos de cavidad suficiente para colocar los trocares de trabajo para seguir con la intervención. Tras la liberación de las adherencias de disecara la región anatómica de trabajo.

En caso de pacientes obesos la colocación de los trocares en los abordajes transperitoneales renales debe de alejarse de la línea media, posicionando generalmente la puerta de entrada inicial por fuera de



FIG URA 1: La inserción del trócar debe de seguir la dirección del órgano diana. la línea externa de los rectos anteriores cuando el paciente ha sido colocado en decúbito lateral y su abdomen se "desplaza" parcialmente hacia la línea media. A su vez los trocares de trabajo se deben ubicar más cerca de la región renal pues la longitud estándar de los instrumentos puede que no nos permita llegar a la región craneal del campo quirúrgico y dificultarnos el abordaje del polo superior renal y la disección de la glándula suprarrenal. Al igual que en pacientes sin sobrepeso la colocación de las distintas puertas de entrada debe seguir la dirección de la línea de trabajo del riñón, pues si esto no se cumple estaremos permanentemente trabajando contra la presión que ejerce la pared abdominal sobre el trocar y reconduciendo continuamente la dirección del mismo (Figura 1).

En casos de obesidad mórbida es preceptivo proveerse de trocares especiales diseñados al uso que son mas largos y que nos permitirán trabajar más cómodamente. También a consejamos la colocación del

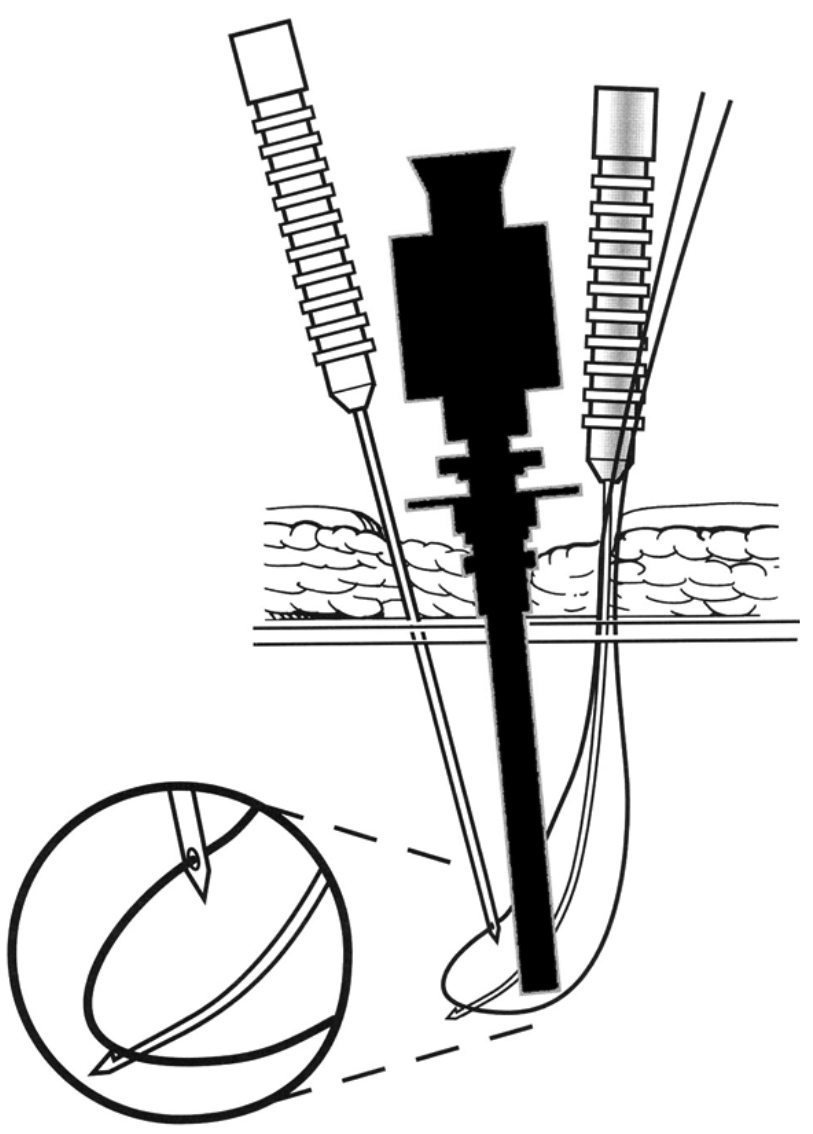

FIG URA 2: Dispositivo de Carter-Thomason. 
trocar inicial bajo visión directa, aunque un abordaje ciego directo mediante aguja de verres o trocar es factible en manos expertas.

La lesión de los vasos epigástricos es más frecuente en la colocación de los trocares en cirugía pelviana que en el abordaje renal. El consejo que se puede dar para evitar su lesión es que el trocar de trabajo que se coloca en las fosas iliacas izquierda 0 derecha según el lado problema, se realice tras la maniobra de transiluminar con la óptica la pared abdominal para detectar los vasos que discurren a su través. Esto nos evitará muchas lesiones y pérdida de tiempo quirúrgico.

La lesión venosa inadvertida puede que no se manifieste pues puede quedar coaptada por la presión del trocar durante el tiempo que dura la intervención, aunque al extraer el trocar en ocasiones reinicia su sangrado, por ello al finalizar la intervención un paso muy importante es la retirada de los trocares, que debe realizarse bajo control óptico, y disminuyendo la presión intrabdominal lentamente para poder dejar de manifiesto pequeñas lesiones venosas que se hallan ocluidadas por la presión intrabdominal.
Si de forma desafortunada lesionamos un vaso arterial se nos evidenciara durante el transcurso de la intervención observándose un goteo persistente por la pared del trocar que nos obligará a abordar el problema de inmediato. Para el tratamiento de las lesiones venosas de poca importancia si estas se hallan en la porción más interna de la pared muscular pueden solucionarse mediante la coagulación bipolar de la misma bajo control óptico. En otros casos tras la extracción del trocar y la colocación de una sonda de Foley y control bajo visión, puede solucionar un sangrado venoso. En caso contrario o de lesiones arteriales se aconseja la practica de un cierre primario de la incisión del trocar mediante la colocación de un punto de sutura que se rea lizará bajo control endoscópico ya sea mediante la ayuda de una aguja de Reverdin 0 mediante distintos dispositivos de cierre fascial comercializados por distintas casas comerciales (Endoclose, Aguja de Maciol, Dispositivo de Carter-Thomason) que se basan en la introducción de una sutura montada en una aguja que permite su liberación intrabdominal bajo visión y la recuperación de la misma tras la punción de la aguja en el borde contrario de la herida para así cerrar el agujero e incluir al vaso en dicha sutura (Figura 2). 